Review

\title{
Minichromosomes: Vectors for Crop Improvement
}

\author{
Jon P. Cody ${ }^{\dagger}$, Nathan C. Swyers ${ }^{\dagger}$, Morgan E. McCaw ${ }^{\dagger}$, Nathaniel D. Graham ${ }^{\dagger}$, Changzeng Zhao \\ and James A. Birchler *
}

Division of Biological Sciences, University of Missouri, 311 Tucker Hall, Columbia, MO 65211-7400, USA; E-Mails: joncody@mail.missouri.edu (J.P.C.); ncs89f@mail.missouri.edu (N.C.S.); mem7b6@mail.missouri.edu (M.E.M.); ndgraham@mail.missouri.edu (N.D.G.); zhaoc@missouri.edu (C.Z.)

$\dagger$ These authors contributed equally to this work.

* Author to whom correspondence should be addressed; E-Mail: BirchlerJ@Missouri.edu; Tel.: +1-573-882-4905; Fax: +1-573-882-0123.

Academic Editor: Gareth Norton

Received: 15 May 2015 / Accepted: 24 June 2015 / Published: 6 July 2015

\begin{abstract}
Minichromosome technology has the potential to offer a number of possibilities for expanding current biofortification strategies. While conventional genome manipulations rely on random integration of one or a few genes, engineered minichromosomes would enable researchers to concatenate several gene aggregates into a single independent chromosome. These engineered minichromosomes can be rapidly transferred as a unit to other lines through the utilization of doubled haploid breeding. If used in conjunction with other biofortification methods, it may be possible to significantly increase the nutritional value of crops.
\end{abstract}

Keywords: minichromosomes; biofortification; B chromosomes; telomere truncation; BIBAC; genetic engineering; haploid induction

\section{Introduction}

While efforts to reduce global hunger have been successful, one in nine humans still suffer from malnourishment [1]. Such a statistic is not exclusively dependent on plant nutritional content, but arises from compounded factors in food security, with developing countries being greatly impacted [1]. 
Many of these developing regions depend on few staple crops for full nutrient intake, which often lack important dietary components, such as essential amino acids, carbohydrates, and minerals [2]. With limited options and access to nutritional supplements, many individuals in these countries suffer from micronutrient malnutrition [1]. Utilization of biofortification strategies may not be the "solve all" answer to global hunger problems. However, these techniques not only offer an opportunity to significantly reduce malnourishment in developing regions, but improve human health worldwide.

Biofortification aims to improve or supplement crop nutritional content through fertilizer application, conventional breeding, and/or genetic engineering [2]. The technique to be used would depend on the specific micronutrient being manipulated; however, complementary utilization of strategies could enhance nutritional output [3]. Inexpensive and simple fertilization strategies are not effective in most scenarios because of several disadvantages including differential effects on crop variants, inability to target edible plant components, and the fact that this approach only modulates the minerals and not the genotype [4]. Conventional breeding is an alternative technique that utilizes favorable characteristics that exist in natural variants and introduces them into commercial lines. The conventional breeding strategy, however, is time consuming and limited by genetic resources that are available $[2,4]$. Alternatively, genetic engineering/biotechnology, is a powerful tool that can be used to directly manipulate the genetic code of specific crop variants to alter metabolic processes or increase mineral uptake [4].

In 1983, researchers demonstrated that an isolated gene fragment could be transformed into a plant species [5-8]. Since then, biotechnology has been making significant contributions to several fields of scientific study, enabling timely alterations to genetic codes without lengthy introgression processes. Additionally, genetic engineering expands the possibilities that are available with conventional breeding by not relying on natural variations, and allowing increased control over gene expression with diverse promoters that are publically available. Because gene delivery is accomplished through either Agrobacterium mediated transformation or particle bombardment, transgene integration is random, which creates a number of limitations with this technology. Notable limitations include, but are not limited to: (1) disruption of endogenous gene function; (2) affected expression from regulatory elements; (3) difficulty of separation from closely associated genes; and (4) inefficient recovery of multiple transgenic events in each successive generation [9].

With many metabolic processes and nutrient accumulation mechanisms requiring multiple gene products, current genetic engineering methods are not an efficient strategy to accomplish the goals of nutrient accumulation in plants. Minichromosome technology, if applied to this problem, offers a unique solution to these limitations through the creation of an autonomous element that works as a platform for transgene stacking and can be transferred efficiently to subsequent generations [10]. Coupled with doubled haploid breeding, transfer of these engineered chromosomes into other varieties could be expedited, allowing rapid analysis of gene aggregates in several crop lines. Here, the basics of minichromosome technology are discussed in the hope of illustrating the potential utility in a number of such applications. 


\section{Discussion}

\subsection{Structure Overview}

In order for minichromosomes to function properly, they must contain the necessary components required to be successfully propagated during cell division. All eukaryotic chromosomes must contain a centromere for kinetochore formation, origins of replication to maintain proper chromosome numbers during cell division, and telomeres to protect the chromosome ends from degradation. Interestingly, in plants, the telomere is the only component that can be synthesized, as the centromere is epigenetic in nature, and the origin of replication has not been elucidated. As a result, minichromosomes must be created by utilizing endogenous centromeres and telomeres in a process known as the top-down method.

\subsection{Centromeres}

The centromere is required in order for chromosomes to segregate properly during cell division. The region is responsible for recruitment of kinetochore proteins, and the ultimate attachment of the chromosome to microtubules for proper movement through the cell cycle. While some organisms only require a short sequence to form a centromere, those of plants are much more complex. Plants have regional centromeres, which are composed of satellite repeats, and can vary widely in number between species and even chromosomes [11-13]. CENH3, the histone H3 variant of plants typical of active centromeres, associates with this repeat region [14]; however, studies have shown that the entire region may not interact [14]. Many plant centromeres are additionally interspersed with retrotransposon sequences, in maize known as CRM elements [15]. CRM elements and satellite repeats both interact with CENH3 and are found throughout the centromere region [14,16]. Not all plant centromeres rely on such repeat regions; wheat, for example, does not possess any tandem repeats within its centromere [17].

Despite most centromeres within a genome containing similar sequences, multiple studies have found that new centromeres can form in regions that are unique in sequence structure [18-20]. Additionally, active centromeres that are formed over repeat regions can be inactivated, as was shown by the recovery of structurally dicentric B chromosomes in maize [21]. The realization that both centromere inactivation and de novo formation occur regularly illustrates an epigenetic component to centromere specification rather than a determination by the DNA sequence [22-24]. As a result, simply including centromeric repeats on a potential minichromosome construct is unlikely to induce kinetochore formation when introduced into a cell.

\subsection{Origins of Replication}

The timely duplication of the genome is an important step in the cell reproduction process. DNA synthesis is strictly regulated by a variety of mechanisms that determine the time and location of replication fork assembly [25]. The number of replication origins in a genome is mostly dependent on chromosome size [25]. In most prokaryotes, there exists only one origin of replication on the circular chromosome. Replication origins have been identified in many prokaryotes and there are even tools for 
predicting their locations. Eukaryotes, generally, have many origins of replication that assemble at different times, which allows for the replication of the large linear eukaryotic chromosomes.

$S$. cerevisiae is the only eukaryote where the replication origin has been identified. Neither a specific location nor consensus sequence has been found for origins of replication in any other yeast strains, nor in higher eukaryotes [25]. In metazoans there are many origins of replication, which are not all active in every cell [25]. With regard to minichromosomes, this situation eliminates any concern for specifically identifying replication origin constitution.

\subsection{Telomeres}

The telomere of the chromosome is a sequence repeat, and accompanying protein complex, that protects the end from damage or chromosome end-to-end fusions. While the majority of the telomere is a heterochromatic region, the extreme chromosome terminus is protected by a single strand overhang that forms a protective loop, known as a G loop [26]. The sequence that confers telomere function is highly conserved, with most plants having a repeat sequence of TTTAGGG that can be extended with the enzyme telomerase [27]. The telomerase is required for telomere extension as DNA polymerase can only extend after a primer template, leading to a loss of sequence at the end of the chromosome during replication. This restriction in synthesis leads to a shorter telomere sequence with each chromosomal replication event. While telomerase has the ability to extend shortened telomeres, telomerase expression varies widely between tissue types, showing little activity in mature tissues [28,29].

For the purposes of minichromosome creation, synthetically produced telomere sequences are introduced with the desired transgene. In most instances, the introduced telomere will be shorter than the endogenous telomere that it replaces. In order to be functional, these repeats must be extended and the end modified to include a $\mathrm{G}$ loop. While the exact process that occurs when a synthetic telomere is introduced is unknown, there are studies that suggest that telomere length is monitored, and can be extended in some organisms when the repeat number is too low [30]. Additionally, studies of Arabidopsis telomeres show that the repeat number can vary between individual chromosomes, and different ecotypes suggesting that the amount of telomere is being actively regulated [31]. As a result, despite minichromosomes not containing the required amount of telomere upon introduction, the plant contains the machinery required to extend the copy number to the essential number.

\subsection{Utilization of B Chromosomes}

B chromosomes are supernumerary chromosomes found in many species of plants. B chromosomes are dispensable. They contain no genes essential to the survival of a plant [32], and show little to no effect on fitness except at high copy number $[33,34]$. B chromosomes are maintained in populations by a drive mechanism. This process consists of nondisjunction of the $\mathrm{B}$ chromosome. This results in more $\mathrm{B}$ chromosomes in the progeny than in the parents. In maize (Zea mays) nondisjunction happens at the second pollen mitosis, which makes the two maize sperm. A plant with one B chromosome produces pollen with sperm containing two B chromosomes or containing none. The sperm with the B chromosomes then preferentially fertilizes the egg cell rather than the polar nuclei. [32]. In rye (Secale cereale) nondisjunction occurs at the first pollen mitosis and the two B chromosomes are directed to 
the generative nucleus resulting in both sperm carrying two B chromosomes [32]. This directed nondisjunction also occurs during the formation of the egg. In maize, two regions are necessary for the nondisjunction of the $\mathrm{B}$ centromere. One is located in the proximal euchromatic region, while the other is at the distal end of the long arm [35]. Both act in trans. In rye, only a region on the distal end of the long arm is necessary for nondisjunction.

$\mathrm{B}$ chromosomes are useful in the creation of an engineered minichromosome because telomere mediated truncation involves the removal of some or most of at least one chromosome arm. If an A chromosome is truncated, this will generally lead to a detrimental monosomic condition, though rare events of minichromosomes with an A chromosome centromere and otherwise normal complement of chromosomes have been reported [36]. Truncating a B chromosome has no detrimental effect on the phenotype of a plant and therefore does not lower the rate of recovery of truncation events.

B chromosomes do not pair or recombine with A chromosomes [32]. This could allow multiple transgenes to be kept together as a unit instead of requiring intensive breeding strategies to stack multiple transgenes in a single plant. Because B chromosomes do not contain any vital genes, there is no chance of linkage of a transgene to an unfavorable allele or knockout of an important gene.

The accumulation mechanism of the B chromosome can be used to increase the copy number of minichromosomes and increase their dosage [37]. Because the truncation of a B chromosome usually removes the distal end of the long arm, it is necessary to have an unreduced B in the background, which can act in trans to restore the nondisjunction property.

\subsection{Alternative Methods for Engineering Minichromosomes}

B chromosomes are not found in some agriculturally important crops. In order to create minichromosomes in these crops an A chromosome must be truncated to provide an active centromere. In a euploid individual the truncation of an A chromosome would be detrimental. For that reason telotrisomic lines are a good candidate for telomere truncation. A telotrisomic individual has a normal complement of chromosomes plus a chromosome consisting of one chromosome arm. Telotrisomics can be found by screening the progeny of a triploid plant for trisomic individuals. From trisomics, centromere misdivision events, or splitting, can produce telotrisomics. A complete set of telotrisomics was developed for rice [38].

Aneuploid plants are less vigorous than euploid; generally the less of the genome for which a plant is aneuploid, the less negative the phenotype. Optimally, a minichromosomes should contain as little of the A genome as possible. Telotrisomics are good candidates for creating minichromosomes because they require only one chromosome arm truncation to remove much of the genic region from the A chromosome for which they are trisomic and relieve the aneuploid phenotype.

The effects of aneuploidy can also be lessened in a higher ploidy background. In maize, a truncated A chromosome was rescued in a spontaneous tetraploidy event [39]. Tetraploid plants have also been used for A chromosome truncation in Arabidopsis thaliana [40] and barley (Hordeum vulgare) [41]. The truncated chromosome can then be transferred to a diploid background by successive crosses of the tetrapliod by a diploid to produce a triploid and again by a diploid to recover the truncated minichromosome as an extra entity in a diploid background [39]. 


\subsection{Stability in Plants}

Minichromosomes derived from the $\mathrm{B}$ chromosome of maize are heritable, but transmitted at varying frequencies. Many minichromosomes fail to pair by metaphase 1 of meiosis. Other irregularities such as early sister chromatid separation and lagging chromosomes during division have also been observed. These meiotic abnormalities appear to be correlated with minichromosome size [42]. Nondisjunction can also occur in somatic tissues, especially at high minichromosome copy number. When mitotic metaphase cells were examined in maize root meristems, B chromosome and minichromosome copy number varied between cells when high numbers were present [37].

Stable transmission of minichromosomes can be achieved in multiple ways. Firstly, larger $\mathrm{B}$ minichromosomes tend to pair better and behave more normally in meiosis than smaller B-derived minichromosomes. A minichromosome derived from a truncation of only the distal tip of a B chromosome will behave like an A chromosome if there are two copies to allow pairing and there are no normal B chromosomes present [42]. To improve transmission of smaller, less stable minichromosomes or minichromosomes in a species without B chromosomes, a gametophytic selection mechanism has been proposed. Cytoplasmic male sterile (cms) plants have a mitochondrial mutation which causes pollen abortion. A nuclear restorer of fertility (Rf) gene prevents pollen abortion in cms plants [43]. Inserting Rf onto a minichromosome would allow any pollen grains with a minichromosome to develop, while any pollen grains without a minichromosome would abort [44].

\subsection{Transgene Expression}

Random integration of genetic fragments can lead to varying levels of expression, due to possible silencing events that occur at different loci throughout the host genome. This phenomenon, coupled with repressed transcription of repeated or homologous sequences, is an obstacle in the field of genetic engineering [45,46]. Artificial minichromosome technology offers an autonomous platform for sequential transgene integration events at a specific locus that has been demonstrated to successfully express genes at detectable levels. However, utilization of repeated genetic elements needs to be avoided when designing minichromosomes due to possible homologous gene silencing events [46]. While still in preliminary stages of development, the use of synthetic promoters will be a valuable tool in avoiding sequence repeats [47]. As both artificial minichromosomes and synthetic promoter technologies progress in parallel, complementary use of both systems will work synergistically to expand limits of genetic engineering and avoid possible silencing events that occur from sequence homology.

\subsection{Creating Large Minichromosomes with BIBACs}

Genetic engineering has, so far, focused mostly on introducing single or a few genes into crops. While these crops have been successful, more complex traits such as improved nutrition will rely on stacking multiple genes [48]. Thus far, introduction of multiple genes has relied on complex breeding to combine separately introduced genes, or co-introducing multiple genes during one transformation. As a result, the ability to introduce multiple traits on one T-DNA is preferred. 
Vectors capable of carrying large $(>300 \mathrm{~kb})$ DNA fragments, known as bacterial artificial chromosome (BAC) vectors, were first created for use in E. coli [49]. Shortly after, the vectors were modified to have the ability to be maintained in Agrobacterium tumefaciens as transformation vectors [50]. The result, known as binary bacterial artificial chromosome (BIBAC) vectors, have been shown to transfer large intact DNA fragments in a variety of different plant species [50-52]. By including telomere sequences near the right border of a BIBAC vector, it should be possible to create minichromosomes while introducing large DNA fragments. These minichromosomes would allow multiple traits to be introduced at a single locus, and would only require a single selection for the entire transgene array.

\subsection{In Vivo Modification of Minichromosomes}

As previously stated, traditional genome manipulation tools, such as Agrobacterium mediated transformation and biolistic bombardment, impose a number of notable limitations. Introducing multiple traits into a single background will require several rounds of transformation, which can be labor intensive and time consuming. Through the utilization of genome targeting mechanisms, minichromosome technology offers a novel solution to these constraints. Precise targeting is derived from a number of site-specific recombination systems that add, remove, or modify specific genetic elements, allowing the assembly of large gene aggregates in a regulated manner.

A diverse number of site-specific recombination systems are publically available for research purposes [53,54]. While unique in sequence recognition, all systems follow the same basic steps: (1) Expressed recombinase enzymes bind to respective sites; (2) Bound enzymes form a synaptic complex, with sites assembled in a parallel orientation; (3) Recombinases catalyze the crossover and fusion of genetic material between recognized cassettes; (4) Disassembly of the synaptic complex [53]. The outcome of the recombination reaction is dependent on location and orientation of the recognition sites. Integration, inversion, and excision of specific genetic elements are all possible, so careful planning of experimental design needs to be carried out to ensure anticipated outcomes. It should be noted that all enzymes fall under a particular sub-category of recombinases that reflect the nature of the system [54]. These categories differ in a number of characteristics, including size and directionality [54]. Such a collection of recombinases can be exploited for the purpose of large-scale genetic manipulations, specifically the creation of artificial minichromosomes.

Regulation of recombinase expression is of most importance in assembling gene aggregates on minichromosomes. Several strategies to control the timing and duration of recombinase activity have been demonstrated [55]. With sexually propagating plants, backgrounds that are actively expressing a recombinase enzyme can be bred with lines containing a minichromosome that is to be modified. The recombinase gene could simply be crossed out in the next generation, leaving a background that only contains a modified minichromosome. Organisms that reproduce vegetatively, or require lengthy germination cycles, could favorably use tactics that transiently express the recombinase enzyme. An example of one of these methods is the auto-excision strategy. Under the control of an inducible promoter, recombinases can be activated by a number of factors, including heat-shock, chemical, or developmental cues [55]. If flanked by respective recombination sites, induction of an inducible 
promoter will lead to expression of the recombinase, followed by its removal via intra-molecular recombination $[54,55]$.

Taken together, minichromosome construction will require strategic utilization of several site-specific recombination systems. Each respective recognition site is assembled in a specific orientation to allow integration of gene fragments and removal of selectable markers in a successive manner [56]. The proposed transgene stacking strategy exploits recombinase directionality, which enables recombination site and selectable marker recycling with each round of integration [56]. Before this process can be initiated, however, the minichromosome generated must contain a specific sequence that is to be acted upon by a well-characterized recombination system for the purpose of integration. A background that contains a minichromosome, and actively expresses a recombinase, will be introduced to a gene fragment that contains gene(s) of interest, recombination sites, and a selectable marker. The expressed recombinase will form a synaptic complex between its respective recognition sites on the minichromosome and the gene fragment, initiating strand exchange and integration. Transgene orientation upon insertion is predictable and unidirectional, due to the nature of the specific recombination system used. The selectable marker, now bordered by recombination cassettes, is used to identify a successful integration event. Depending on the method of reproduction, a different site-specific recombination system, for the purpose of excision, will be activated through genetic crosses or transient expression. This recombinase will identify the flanking recognition sequences and remove the selectable marker, restoring a single recombination site to be used in the next round of modifications.

\subsection{Transfer of Minichromosomes with Haploid Induction}

It may be possible to transfer minichromosomes to multiple lines rapidly through haploid induction. If a haploid inducing line containing a minichromosome is used to produce haploid embryos it should be possible to recover haploids with one or more minichromosomes as follows. In maize, high haploid induction rate lines have been derived from a line called Stock 6 [57], and its derivatives. When used as a pollen donor Stock 6 derived haploid inducer lines produce maternal haploids at a high rate. Many groups have noted the transfer of markers from the inducer line to the maternal haploids [58,59], and the transfer of complete B chromosomes has also been reported [59]. Minichromosomes introduced into the inducer line should be able to be transferred in an analogous fashion.

Once a haploid plant with a minichromosome has been obtained, its ploidy can be doubled by colchicine or high-pressure nitrous oxide gas [60]. These treatments produce diploid sectors in the plant which are fertile and the plant can be self-pollinated. This results in a doubled haploid line, which is completely homozygous. By this method, once a minichromosome is back-crossed into a haploid inducing line, it can be transferred to new lines with just two crosses: One to generate the haploid and one for self-pollination. The resultant progeny will be completely homozygous which is basically not possible if the minichromosome had been back-crossed into the line using a typical introgression scheme. 


\section{Conclusions}

As the population of the world continues to increase, there is a growing need to find innovative ways to utilize the static amount of arable land. Biofortification of crops through conventional breeding and fertilizer application are two strategies to make headway toward improving nutritional value, but genetic engineering offers another strategy for addressing global food security. Gene stacking could allow for complex traits and pathways to be expressed with minimal selective breeding. Minichromosomes could provide a stable and heritable platform for this gene stacking. Because a bottom up approach toward creating an artificial chromosome is not yet possible, the top-down strategy for minichromosome creation is a viable option. B chromosomes, which are present or could be introduced in some crop species, offer a platform for creation of minichromosomes with the advantage that they lack essential genes. Other options as outlined above are also available. Minichromosomes have been shown to reliably express transgenes and to be transmitted from generation to generation. There is potential to make large scale additions to minichromosomes, which could allow for the introduction of multiple genes at once instead of through several transformations. There are now a variety of recombination systems, as well as genome editing technologies, which can be utilized to edit an existing minichromosome and make minichromosomes a truly custom platform. The ability to transfer minichromosomes through haploid breeding would allow for the rapid introduction of minichromosomes to many inbred lines, including those not amenable to transformation, without the need for generations of introgression. For these reasons, minichromosomes could provide a tool for improved biofortification of a variety of crop species.

\section{Acknowledgments}

Research on this topic was funded by National Science Foundation grant IOS-1339198.

\section{Conflicts of Interest}

The authors declare no conflict of interest.

\section{References}

1. FAO; IFAD; WFP. Strengthening the enabling environment for food security and nutrition. In The State of Food Insecurity in the World 2014; FAO: Rome, Italy, 2014.

2. Hirschi, K.D. Nutrient biofortification of food crops. Annu. Rev. Nutr. 2009, 29, 401-421.

3. Bruulsema, T.W.; Heffer, P.; Welch, R.M.; Cakmak, I.; Moran, K. Fertilizing Crops to Improve Human Health: A Scientific Review; International Plant Nutrition Institute: Norcross, GA, USA, 2012.

4. Zhu, C.; Naqvi, S.; Gomez-Galera, S.; Pelacho, A.M.; Capell, T.; Christou, P. Transgenic strategies for the nutritional enhancement of plants. Trends Plant Sci. 2007, 12, 548-555.

5. Bevan, M.W.; Flavell, R.B.; Chilton, M. A chimaeric antibiotic resistance gene as a selectable marker for plant cell transformation. Nature 1983, 304, 184-187.

6. Herrera-Estrella, L.; Depicker, A.; Montagu, V.M.; Schell, J. Expression of chimaeric genes transferred into plant cells using a Ti-plasmid-derived vector. Nature 1983, 303, 209-213. 
7. Fraley, R.T.; Rogers, S.G.; Horsch, R.B.; Sanders, P.R.; Flick, J.S.; Adams, S.P.; Bittner, M.L.; Brand, L.A.; Fink, C.L.; Fry, J.S.; et al. Expression of bacterial genes in plant cells. Proc. Natl. Acad. Sci. USA 1983, 80, 4803-4807.

8. Murai, N.; Kemp, J.D.; Sutton, D.W.; Murray, M.G.; Slightom, J.L.; Merlo, D.; Reichert, N.A.; Sengupta-Gopalan, C.; Stock, C.A.; Barker, R.F.; et al. Phaseolin gene from bean is expressed after transfer to sunflower via tumor-inducing plasmid vectors. Science 1983, 222, 476-482.

9. Yu, W.; Han, F.; Birchler, J.A. Engineered minichromosomes in plants. Curr. Opin. Biotechnol. 2007, 18, 425-431.

10. Gaeta, R.T.; Masonbrink, R.E.; Krishnaswamy, L.; Zhao, C.; Birchler, J.A. Synthetic chromosome platforms in plants. Annu. Rev. Plant Biol. 2012, 63, 307-330.

11. Kanizay, L.; Dawe, R. Centromeres: Long intergenic spaces with adaptive features. Funct. Integr. Genomics 2009, 9, 287-292.

12. Burrack, L.S.; Berman, J. Neocentromeres and epigenetically inherited features of centromeres. Chromosome Res. 2012, 20, 607-619.

13. Kato, A.; Lamb, J.C.; Birchler, J.A. Chromosome painting using repetitive DNA sequences as probes for somatic chromosome identification in maize. Proc. Natl. Acad. Sci. USA 2004, 101, 13554-13559.

14. Zhong, C.X.; Marshall, J.B.; Topp, C.; Mroczek, R.; Kato, A.; Nagaki, K.; Birchler, J.A.; Jiang, J.; Dawe, R.K. Centromeric retroelements and satellites interact with maize kinetochore protein CENH3. Plant Cell 2002, 14, 2825-2836.

15. Wolfgruber, T.K.; Sharma, A.; Schneider, K.L.; Albert, P.S.; Koo, D.; Shi, J.; Gao, Z.; Han, F.; Lee, H.; Xu, R.; et al. Maize centromere structure and evolution: Sequence analysis of centromeres 2 and 5 reveals dynamic Loci shaped primarily by retrotransposons. PLoS Genet. 2009, 5, e1000743.

16. Jin, W.; Melo, J.R.; Nagaki, K.; Talbert, P.B.; Henikoff, S.; Dawe, R.K.; Jiang, J. Maize centromeres: Organization and functional adaptation in the genetic background of oat. Plant Cell 2004, 16, 571-581.

17. Liu, Z.; Yue, W.; Li, D.; Wang, R.; Kong, X.; Lu, K.; Wang, G.; Dong, Y.; Jin, W.; Zhang, X. Structure and dynamics of retrotransposons at wheat centromeres and pericentromeres. Chromosoma 2008, 117, 445-456.

18. Nasuda, S.; Hudakova, S.; Schubert, I.; Houben, A.; Endo, T. Stable barley chromosomes without centromeric repeats. Proc. Natl. Acad. Sci. USA 2005, 102, 9842-9847.

19. Gong, Z.; Yu, H.; Huang, J.; Yi, C.; Gu, M. Unstable transmission of rice chromosomes without functional centromeric repeats in asexual propagation. Chromosome Res. 2009, 17, 863-872.

20. Fu, S.; Lv, Z.; Gao, Z.; Wu, H.; Pang, J.; Zhang, B.; Dong, Q.; Guo, X.; Wang, X.; Birchler, J.A.; et al. De novo centromere formation on a chromosome fragment in maize. Proc. Natl. Acad. Sci. USA 2013, 110, 6033-6036.

21. Han, F.; Lamb, J.C.; Birchler, J.A. High frequency of centromere inactivation resulting in stable dicentric chromosomes of maize. Proc. Natl. Acad. Sci. USA 2006, 103, 3238-3243.

22. Han, F.; Gao, Z.; Birchler, J.A. Reactivation of an inactive centromere reveals epigenetic and structural components for centromere specification in maize. Plant Cell 2009, 21, 1929-1939. 
23. Birchler, J.A.; Gao, Z.; Sharma, A.; Presting, G.G.; Han, F. Epigenetic aspects of centromere function in plants. Curr. Opin. Plant Biol. 2011, 14, 217-222.

24. Henikoff, S.; Furuyama, T. Epigenetic inheritance of centromeres. Cold Spring Harb. Symp. Quant. Biol. 2010, 75, 51-60.

25. Leonard, A.C.; Mechali, M. DNA replication origins. Cold Spring Harb. Perspect. Biol. 2013, 5, doi:10.1101/cshperspect.a010116.

26. Makarov, V.L.; Hirose, Y.; Langmore, J. Long g tails at both ends of human chromosomes suggest a $\mathrm{C}$ strand degradation mechanism for telomere shortening. Cell 2001, 88, 657-666.

27. Fajkus, J.; Fulnecková, J.; Hulanova, M.; Berkova, K.; Ríha, K.; Matyásek, R. Plant cells express telomerase activity upon transfer to callus culture, without extensively changing telomere lengths. Mol. Gen. Genet. 1998, 260, 470-474.

28. Fitzgerald, M.S.; McKnight, T.D.; Shippen, D.E. Characterization and developmental patterns of telomerase expression in plants. Proc. Natl. Acad. Sci. USA 1996, 93, 14422-14427.

29. Kilian, A.; Heller, K.; Kleinhofs, A. Development patterns of telomerase activity in barley and maize. Plant Mol. Biol. 1998, 37, 621-628.

30. Hemann, M.T.; Strong, M.A.; Hao, L.; Greider, C.W. The Shortest Telomere, Not Average Telomere Length, Is Critical for Cell Viability and Chromosome Stability. Cell 2001, 107. 67-77.

31. Shakirov, E.V.; Shippen, D.E. Length Regulation and dynamics of individual telomere tracts in wild-type arabidopsis. Plant Cell 2004, 16, 1959-1967.

32. Jones, N.; Houben, A. B chromosomes in plants: Escapees from the A chromosome genome. Trends Plant Sci. 2003, 8, 417-423.

33. Randolph, L.F. Genetic characteristics of the B chromosomes in maize. Genetics 1941, 26, 608-631.

34. Staub, R.W. Leaf striping correlated with the presence of B chromosomes in maize. J. Hered. 1987, 78, 71-74.

35. Roman, H. Mitotic nondisjunction in the case of interchanges involving the B-type chromosome in maize. Genetics 1947, 32, 391-409.

36. Gaeta, R.T.; Masonbrink, R.E.; Zhao, C.; Sanyal, A.; Krishnaswamy, L.; Birchler, J.A. In vivo modification of a maize engineered minichromosome. Chromosoma 2013, 122, 221-232.

37. Masonbrink, R.E.; Birchler, J.A. Accumulation of multiple copies of maize minichromosomes. Cytogenet. Genome Res. 2012, 137, 50-59.

38. Cheng, Z.; Yan, H.; Yu, H.; Tang, S.; Jiang, J.; Gu, M.; Zhu, L. Development and applications of a complete set of rice telotrisomics. Genetics 2001, 157, 361-368.

39. Yu, W.; Han, F.; Gao, Z.; Vega, J.M.; Birchler, J.A. Construction and behavior of engineered minichromosomes in maize. Proc. Natl. Acad. Sci. USA 2007, 104, 8924-8929.

40. Teo, C.H.; Ma, L.; Kapusi, E.; Hensel, G.; Kumlehn, J.; Schubert, I.; Houben, A.; Mette, M.F. Induction of telomere-mediated chromosomal truncation and stability of truncated chromosomes in Arabidopsis thaliana. Plant J. 2011, 68, 28-39.

41. Kapusi, E.; Ma, L.; Teo, C.H.; Hensel, G.; Himmelbach, A.; Schubert, I.; Mette, M.; Kumlehn, J.; Houben, A. Telomere-mediated truncation of barley chromosomes. Chromosoma 2012, 121, 181-190.

42. Han, F.; Gao, Z.; Yu, W.; Birchler, J.A. Minichromosome analysis of chromosome pairing, disjunction, and sister chromatid cohesion in maize. Plant Cell 2007, 19, 3853-3863. 
43. Chase, C.D.; Gabay-Laughnan, S. Cytoplasmic male sterility and fertility restoration by nuclear genes. In Molecular Biology and Biotechnology of Plant Organelles; Daniell, H., Chase, C.D., Eds.; Springer: Dordrecht, The Netherlands, 2004; Volume 22, pp. 593-621.

44. Birchler, J.; Krishnaswamy, L.; Gaeta, R.; Masonbrink, R.; Zhao, C. Engineered Minichromosomes in Plants. CRC Crit. Rev. Plant. Sci. 2010, 29, 135-147.

45. Ye, F.; Signer, E.R. RIGS (repeat-induced gene silencing) in Arabidopsis is transcriptional and alters chromatin configuration. Proc. Natl. Acad. Sci. USA 1996, 93, 10881-10886.

46. Matzke, A.J.; Matzke, M.A. Position effects and epigenetic silencing of plant transgenes. Curr. Opin. Plant Biol. 1998, 1, 142-148.

47. Liu, W.; Yuan, J.S.; Stewart, C. Advanced genetic tools for plant biotechnology. Nat. Rev. Genet. 2013, 14, 781-793.

48. Halpin, C. Gene stacking in transgenic plants-The challenge for 21 st century plant biotechnology. Plant Biotechnol. J. 2005, 3, 141-155.

49. Shizuya, H.; Birren, B.; Kim, U.; Mancino, V.; Slepak, T.; Tachiiri, Y.; Simon, M. Cloning and stable maintenance of 300-kilobase-pair fragments of human DNA in Escherichia coli using an F-factor-based vector. Proc. Natl. Acad. Sci. USA 1992, 89, 8794-8797.

50. Hamilton, C.M.; Frary, A.; Lewis, C.; Tanksley, S.D. Stable transfer of intact high molecular weight DNA into plant chromosomes. Proc. Natl. Acad. Sci. USA 1996, 93, 9975-9979.

51. Vega, J.M.; Yu, W.; Han, F.; Kato, A.; Peters, E.M.; Zhang, Z.S.; Birchler, J.A. Agrobacterium-mediated transformation of maize (Zea mays) with Cre-lox site specific recombination cassettes in BIBAC vectors. Plant Mol. Biol. 2008, 66, 587-598.

52. Hamilton, C.M.; Frary, A.; Xu, Y.; Tanksley, S.D.; Zhang, H. Construction of tomato genomic DNA libraries in a binary-BAC (BIBAC) vector. Plant J. 1999, 18, 223-229.

53. Grindley, N.D.; Whiteson, K.L.; Rice, P.A. Mechanisms of site-specific recombination. Annu. Rev. Biochem. 2006, 75, 567-605.

54. Wang, Y.; Yau, Y.; Perkins-Balding, D.; Thomson, J.G. Recombinase technology: Applications and possibilities. Plant Cell Rep. 2011, 30, 267-285.

55. Gidoni, D.; Srivastava, V.; Carmi, N. Site-specific excisional recombination strategies for elimination of undesirable transgenes from crop plants. In Vitro Cell. Dev. Biol. Plant 2008, 44, 457-467.

56. Ow, D.W. 2004 SIVB congress symposium proceeding: Transgene management via multiple site-specific recombination systems. In Vitro Cell. Dev. Biol. Plant 2005, 41, 213-219.

57. Coe, E.H. A line of maize with high haploid frequency. Am. Natl. 1959, 93, 381-382.

58. Zhang, Z.; Qiu, F.; Liu, Y.; Ma, K.; Li, Z.; Xu, S. Chromosome elimination and in vivo haploid production induced by stock 6-derived inducer line in maize (Zea mays L.). Plant Cell Rep. 2008, 27, 1851-1860.

59. Zhao, X.; Xu, X.; Xie, H.; Chen, S.; Jin, W. Fertilization and uniparental chromosome elimination during crosses with maize haploid inducers. Plant Physiol. 2013, 163, 721-731. 
60. Kato, A. Chromosome doubling of haploid maize seedlings using nitrous oxide gas at the flower primordial stage. Plant Breed. 2002, 121, 370-377.

(C) 2015 by the authors; licensee MDPI, Basel, Switzerland. This article is an open access article distributed under the terms and conditions of the Creative Commons Attribution license (http://creativecommons.org/licenses/by/4.0/). 